\title{
Urea Mimics Nucleobases by Preserving the Helical Integrity of B-DNA Duplexes via Hydrogen Bonding and Stacking Interactions
}

\author{
Gorle Suresh, Siladitya Padhi, Indrajit Patil and U. Deva Priyakumar* \\ Center for Computational Natural Sciences and Bioinformatics, \\ International Institute of Information Technology, Hyderabad 500032, India
}

*Corresponding Author

Email: deva@iiit.ac.in

Phone: +91-40-6653 1161

FAX: +91-40-6653 1413 

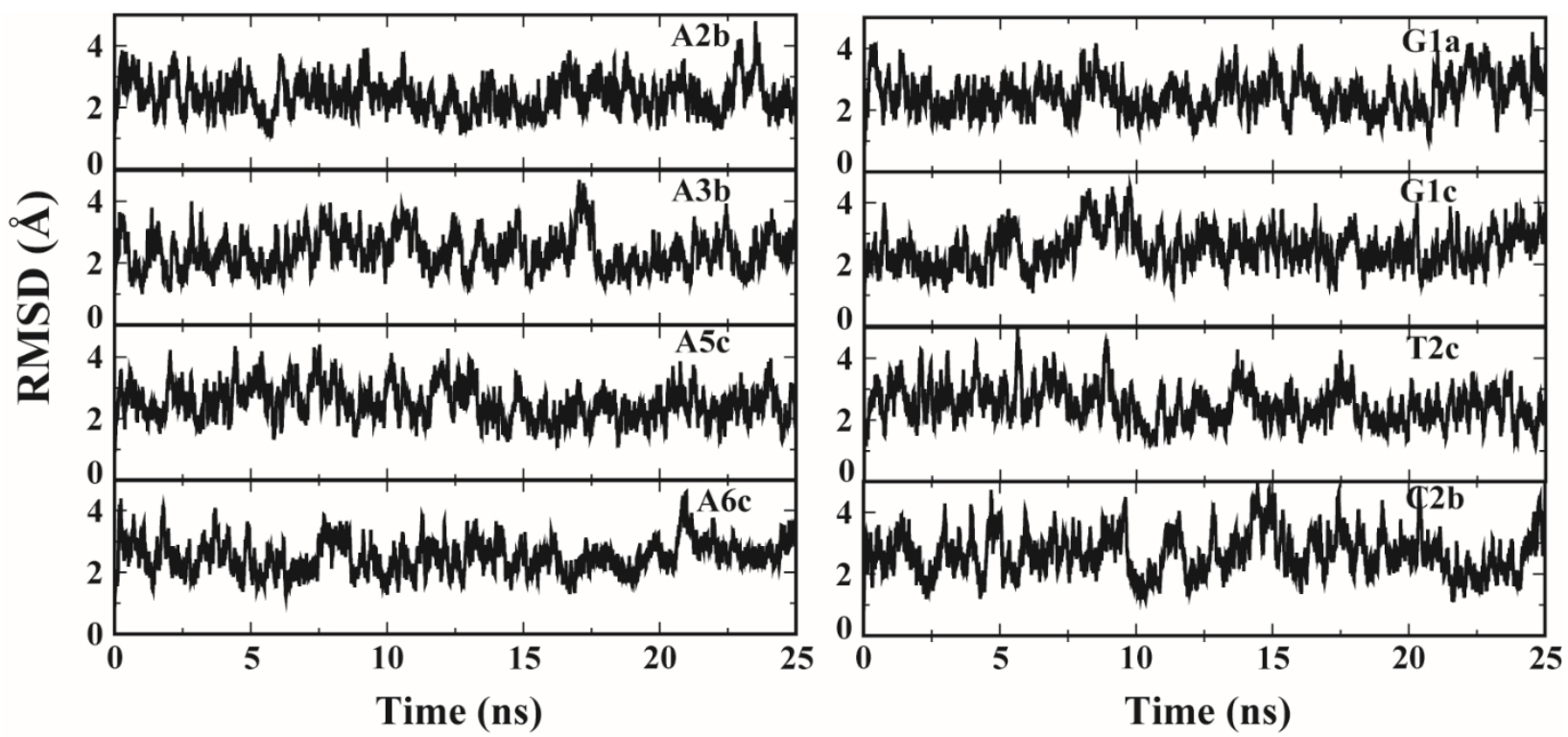

Figure S1: Time series of root mean square deviations (in $\AA$ ) of all the eight urea incorporated BDNA duplexes during the simulations. The respective initial structure was used as a reference to each of the simulation. 

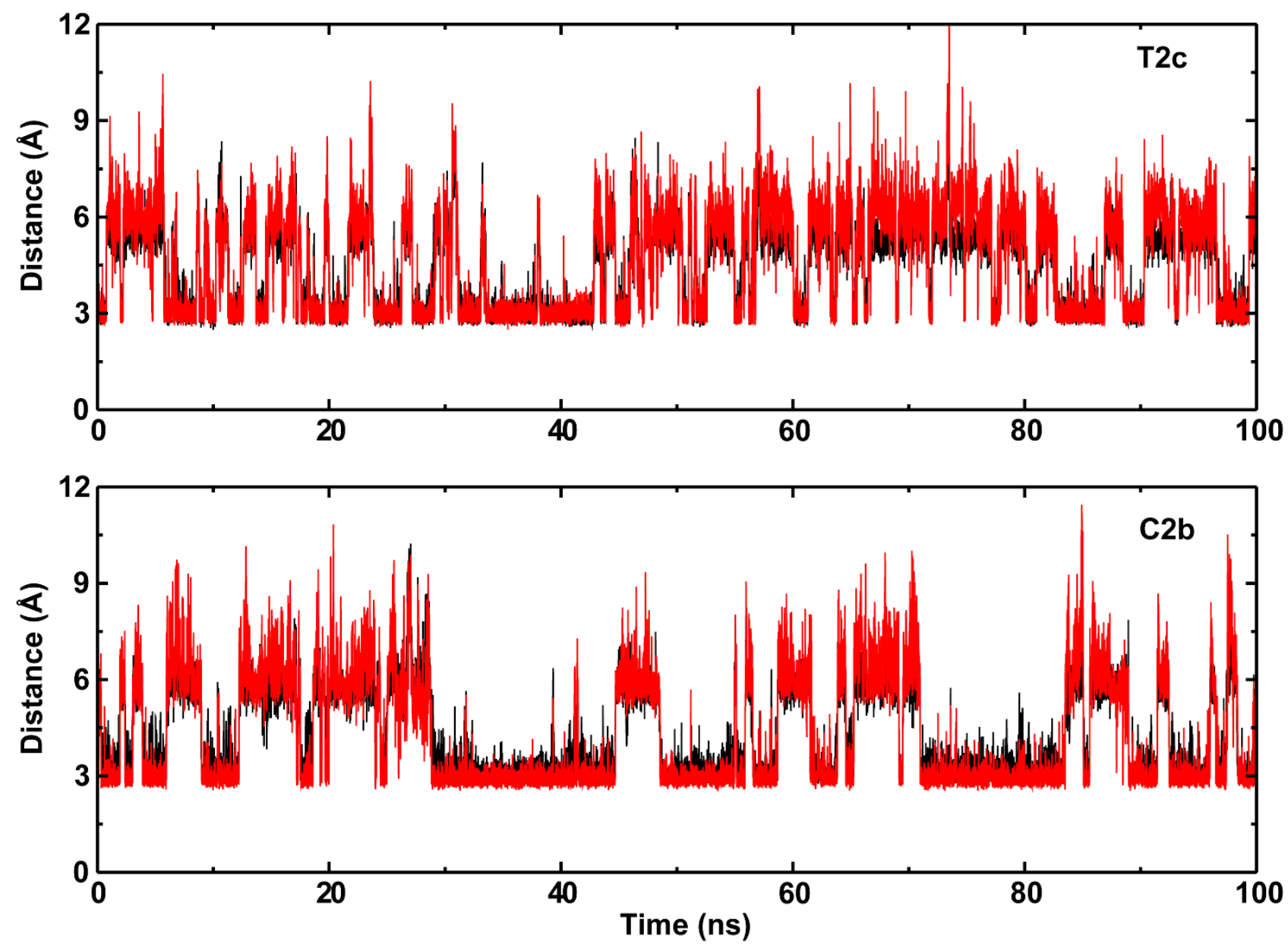

Figure S2: Time series of hydrogen bond distances between pyrimidine and urea bases in T2c and $\mathrm{C} 2 \mathrm{~b}$ during the $100 \mathrm{~ns} \mathrm{MD}$ simulations. 


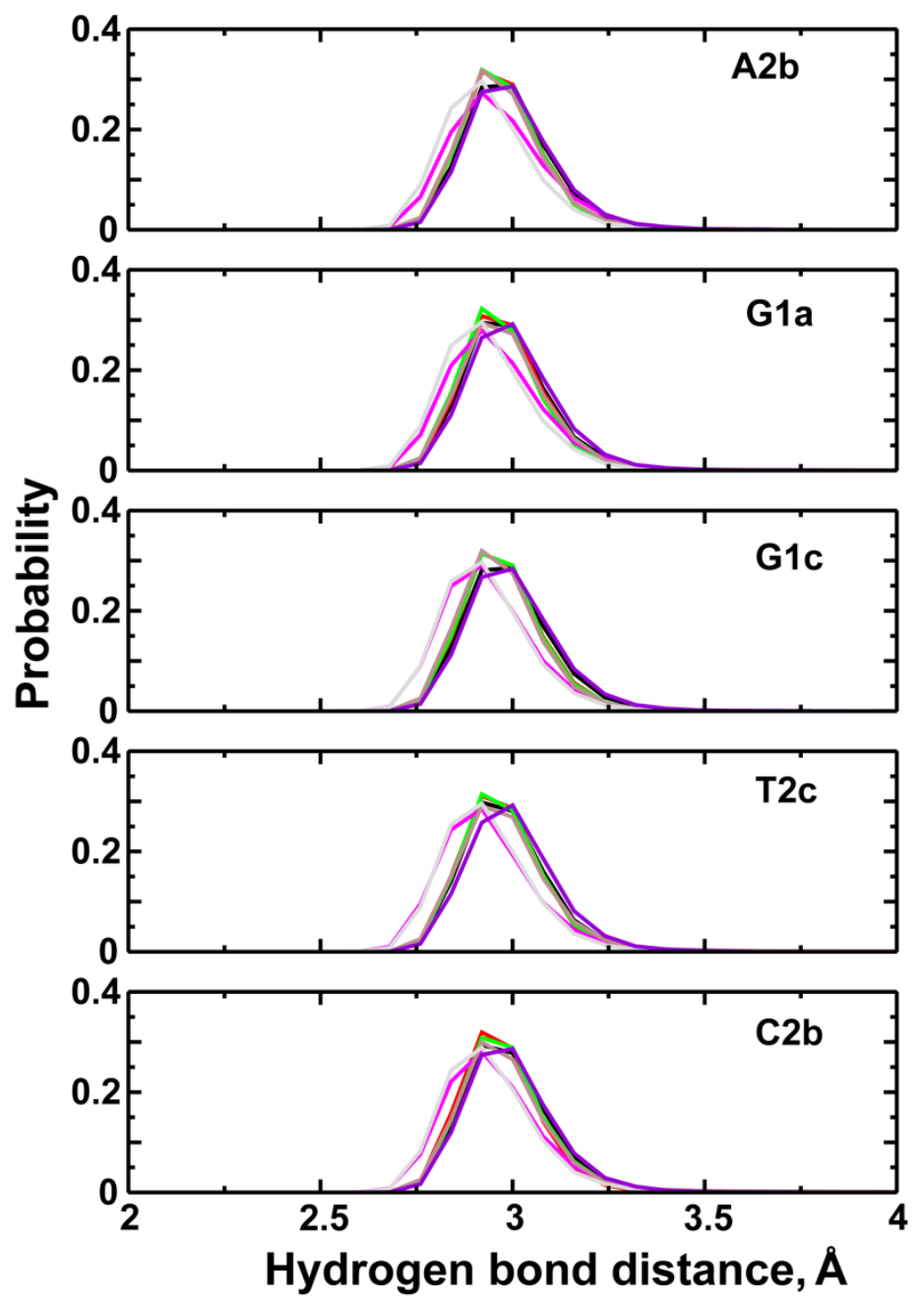

Figure S3: Probability distributions of hydrogen bond distances (in $\AA$ ) of all the base pairs in the urea incorporated B-DNA duplexes during the MD simulations. 
(a)

(b)

G2-C9

C3-G8

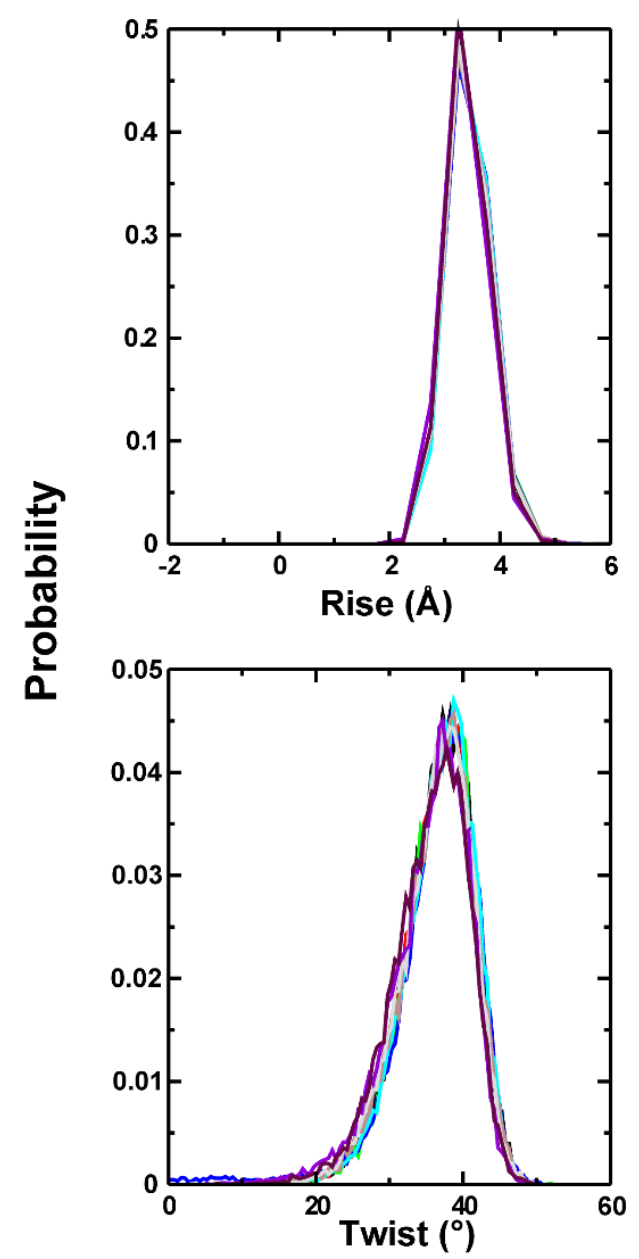

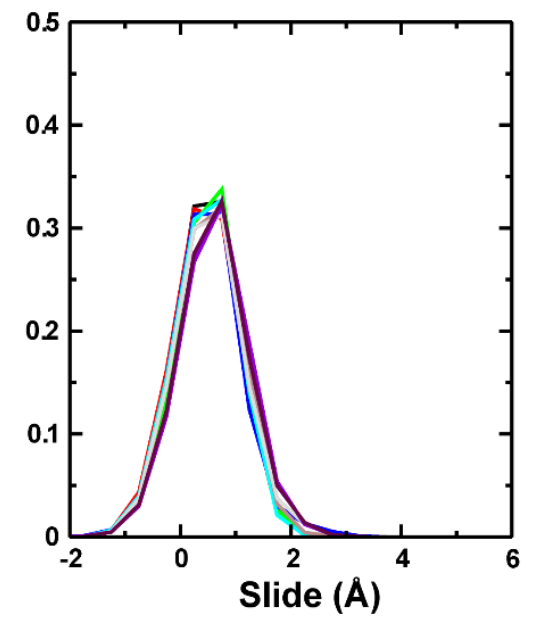
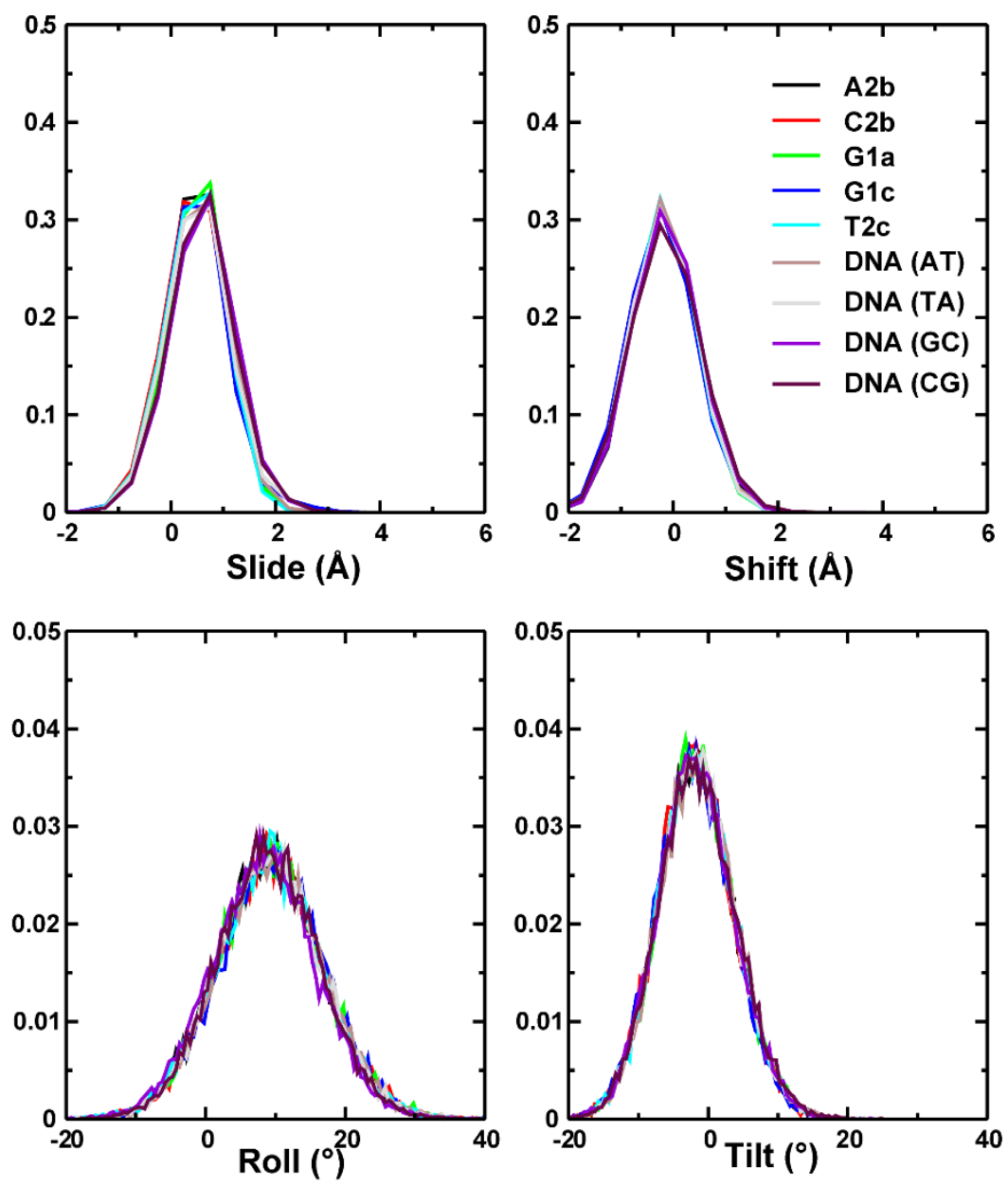

Figure S4: (a) Numbering of the base sequence used during the calculations (b) Probability distributions of base pair step parameters corresponding to base pair step G2C9-C3G8 in all the urea incorporated DNA duplexes. The distributions corresponding to base pair steps in pure DNA are also provided for comparison. All these base pair step parameters were calculated by using Curves+ program. 

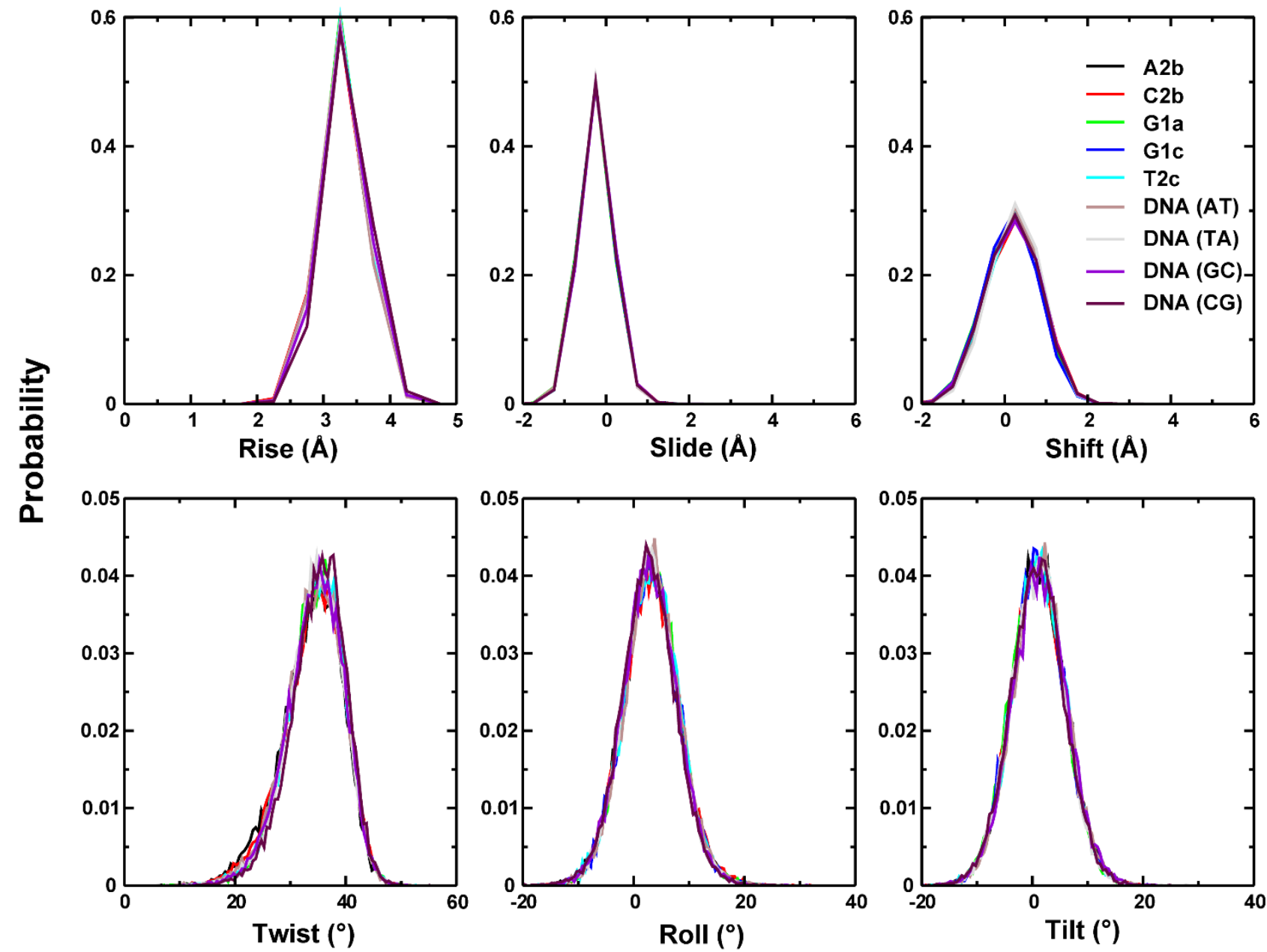

Figure S5: Probability distributions of base pair step parameters corresponding to base pair step C3G8-G4C7 in all the urea incorporated DNA duplexes. The distributions corresponding to base pair steps in pure DNA are also provided for comparison. 
A5-T6
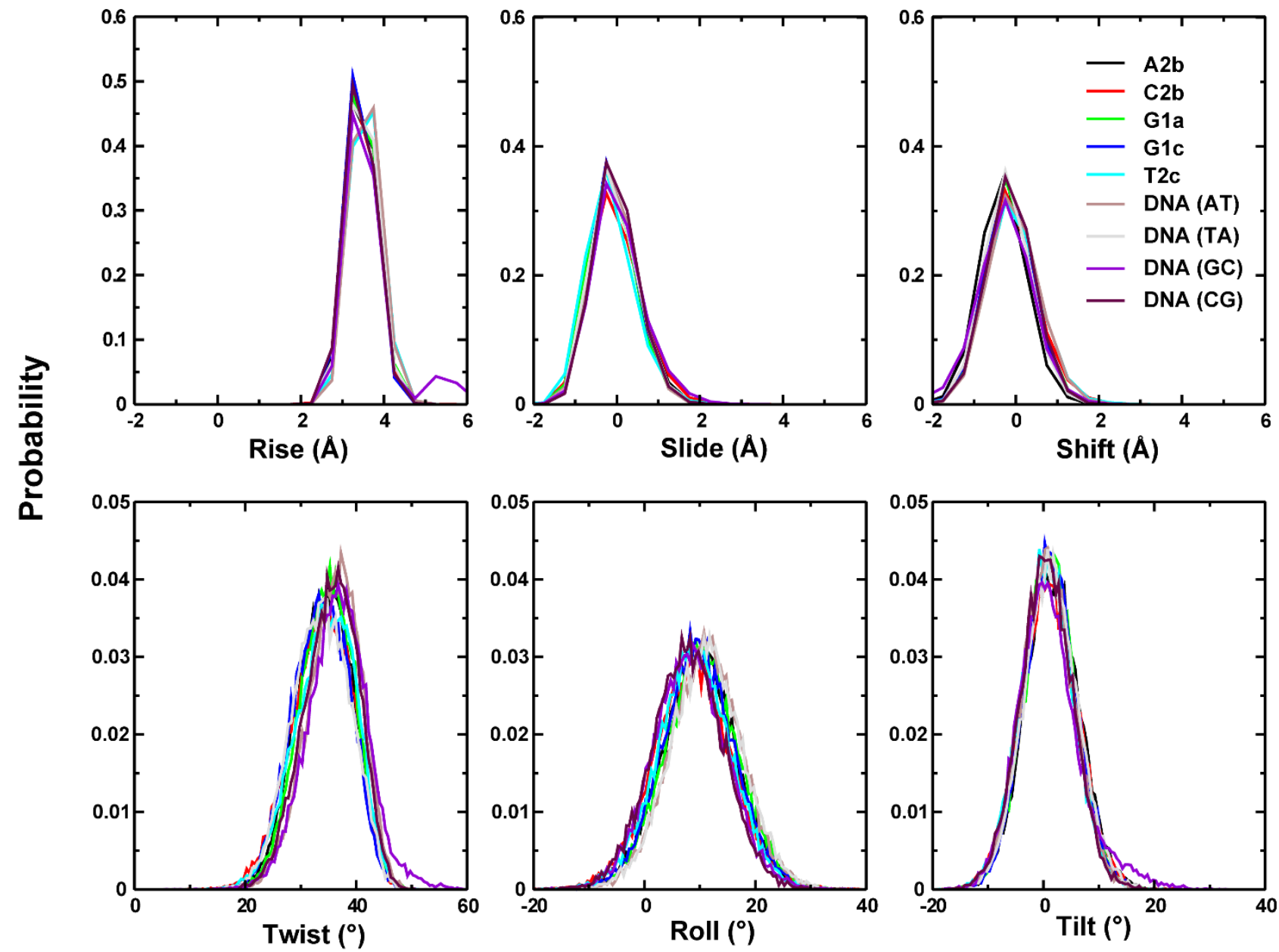

Figure S6: Probability distributions of base pair step parameters corresponding to base pair step G4C7-A5T6 in all the urea incorporated DNA duplexes. The distributions corresponding to base pair steps in pure DNA are also provided for comparison. 
A8-T3
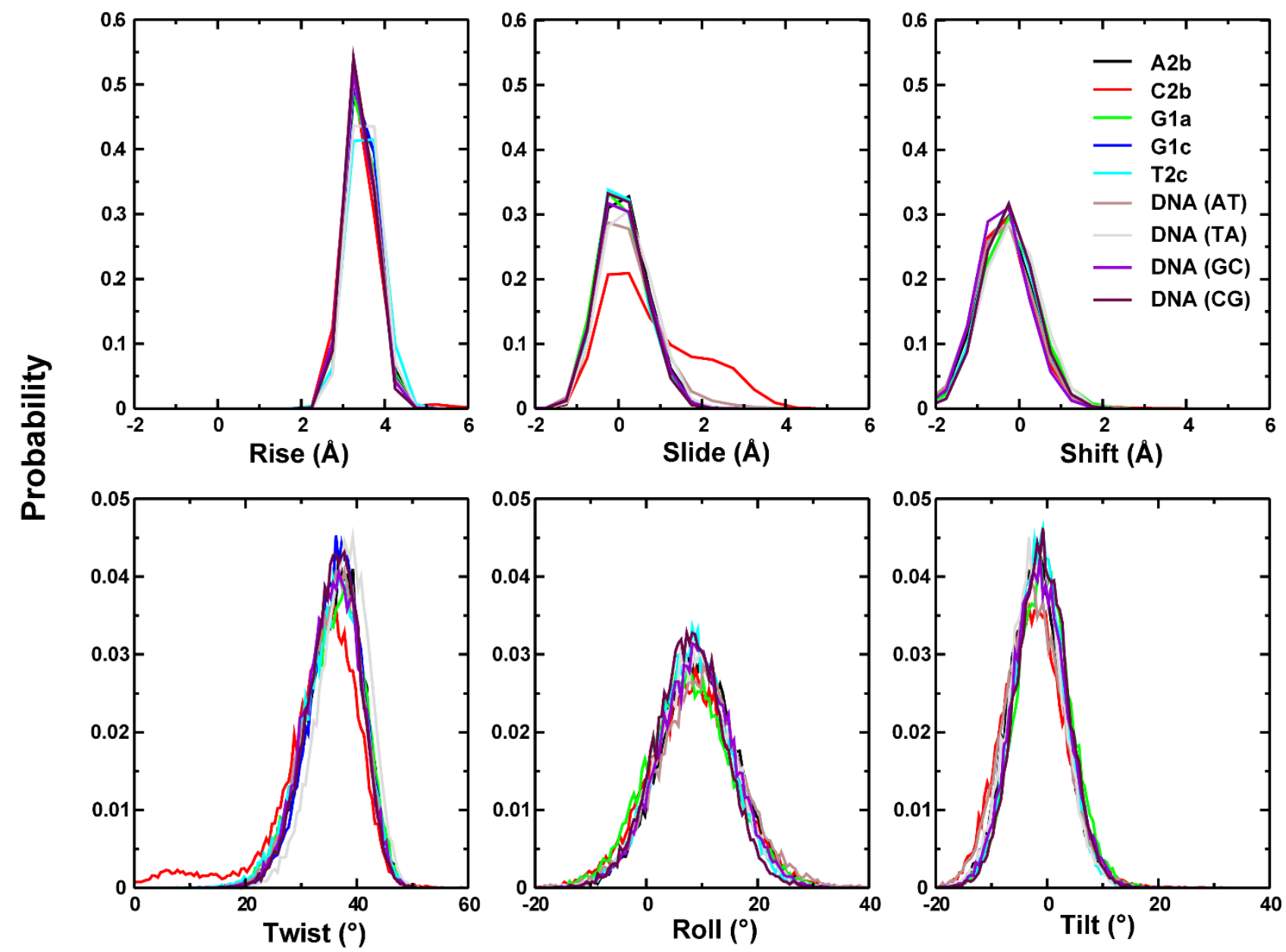

Figure S7: Probability distributions of base pair step parameters corresponding to base pair step C7G4-A8T3 in all the urea incorporated DNA duplexes. The distributions corresponding to base pair steps in pure DNA are also provided for comparison. 
A8-T3

C9-G2
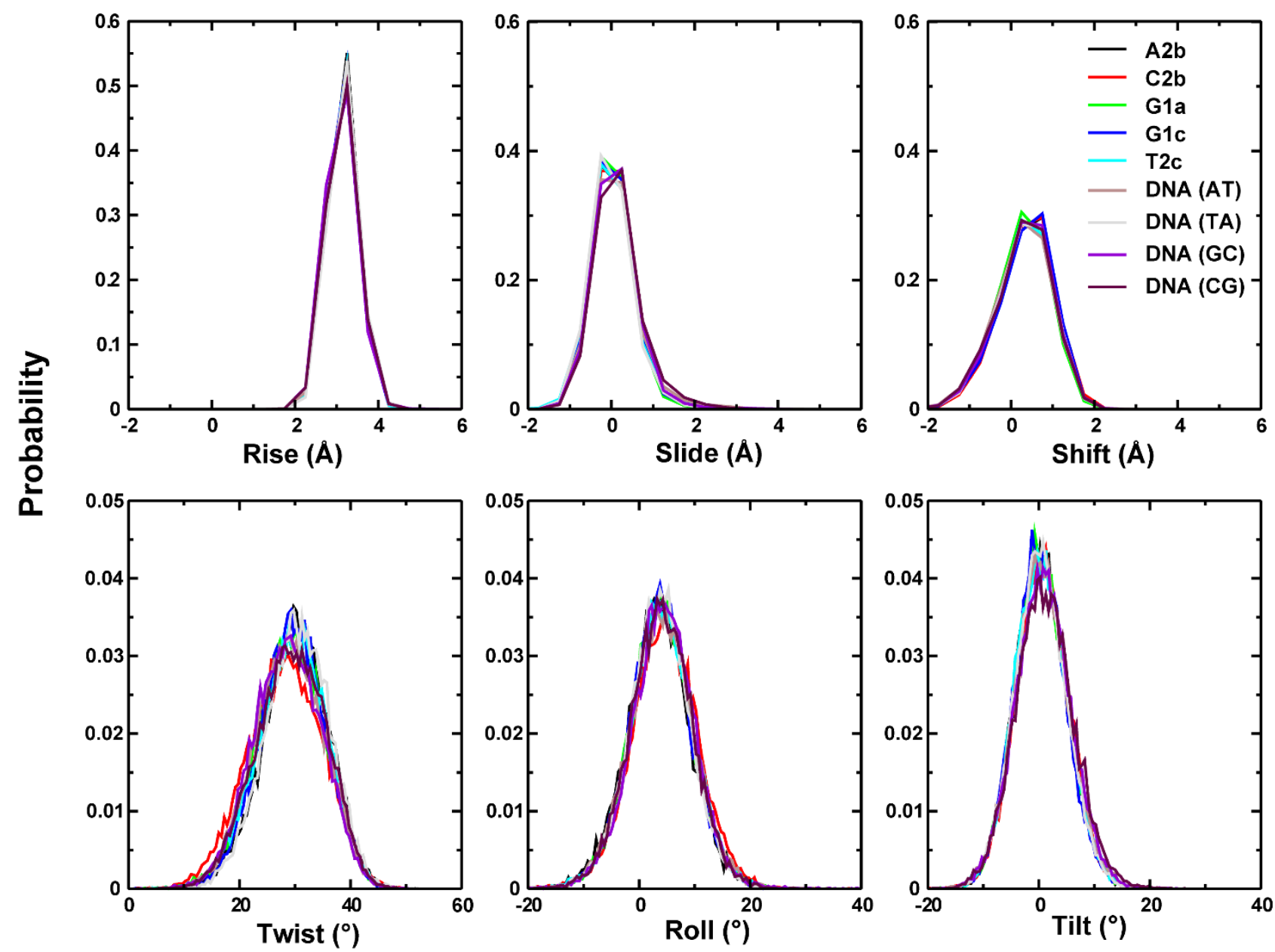

Figure S8: Probability distributions of base pair step parameters corresponding to base pair step A8T3-C9G2 in all the urea incorporated DNA duplexes. The distributions corresponding to base pair steps in pure DNA are also provided for comparison. 


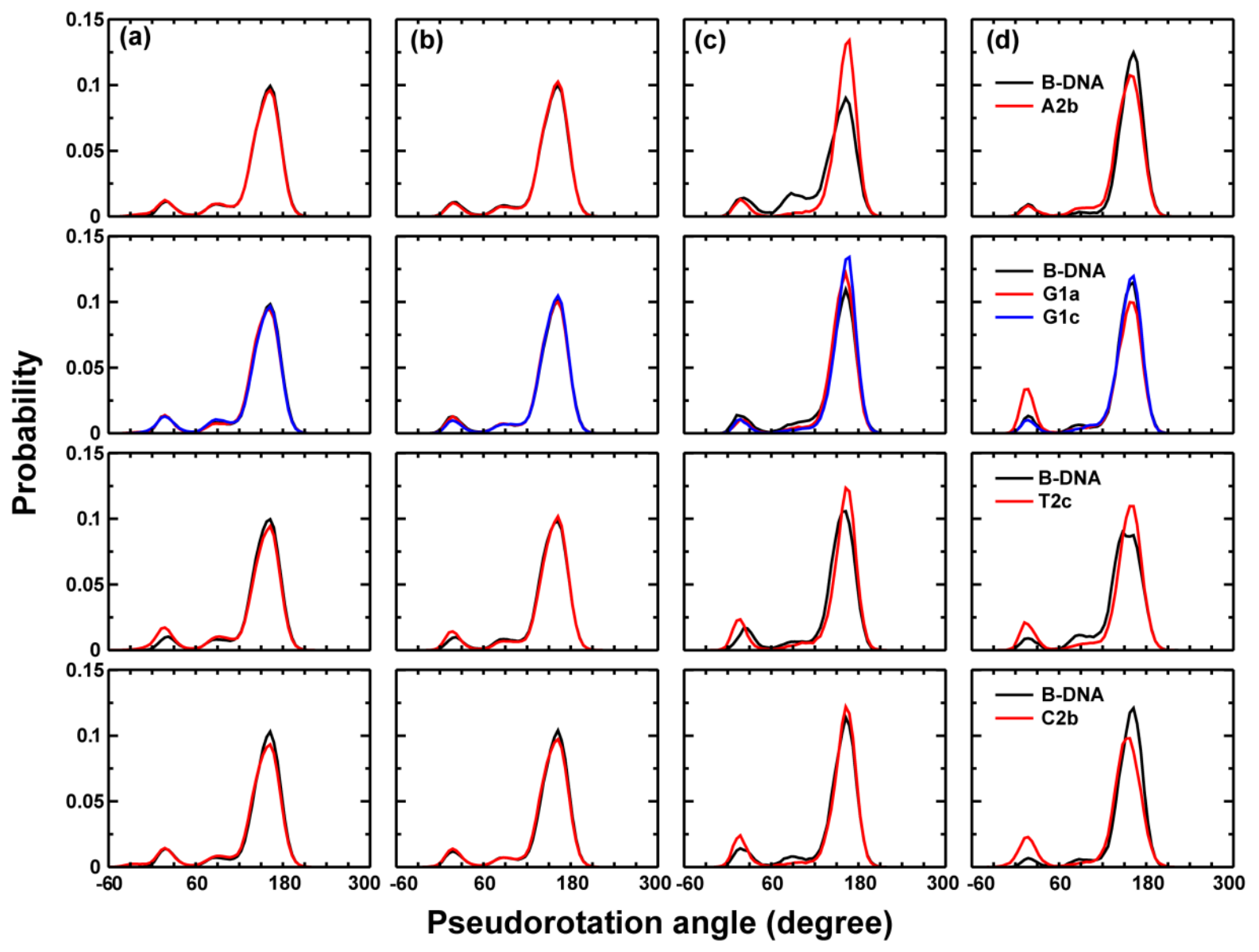

Figure S9: Probability distributions of pseudorotation angles of furanose sugars for the (a) full DNA duplex, (b) full DNA duplex without urea-nucleobase pair, and (c, d) neighbour base pairs in all the urea incorporated DNA duplexes. The pseudorotation angles corresponding to the furanose sugars of typical B-DNA duplexes are shown in black while the urea incorporated DNA duplexes are shown in red. 

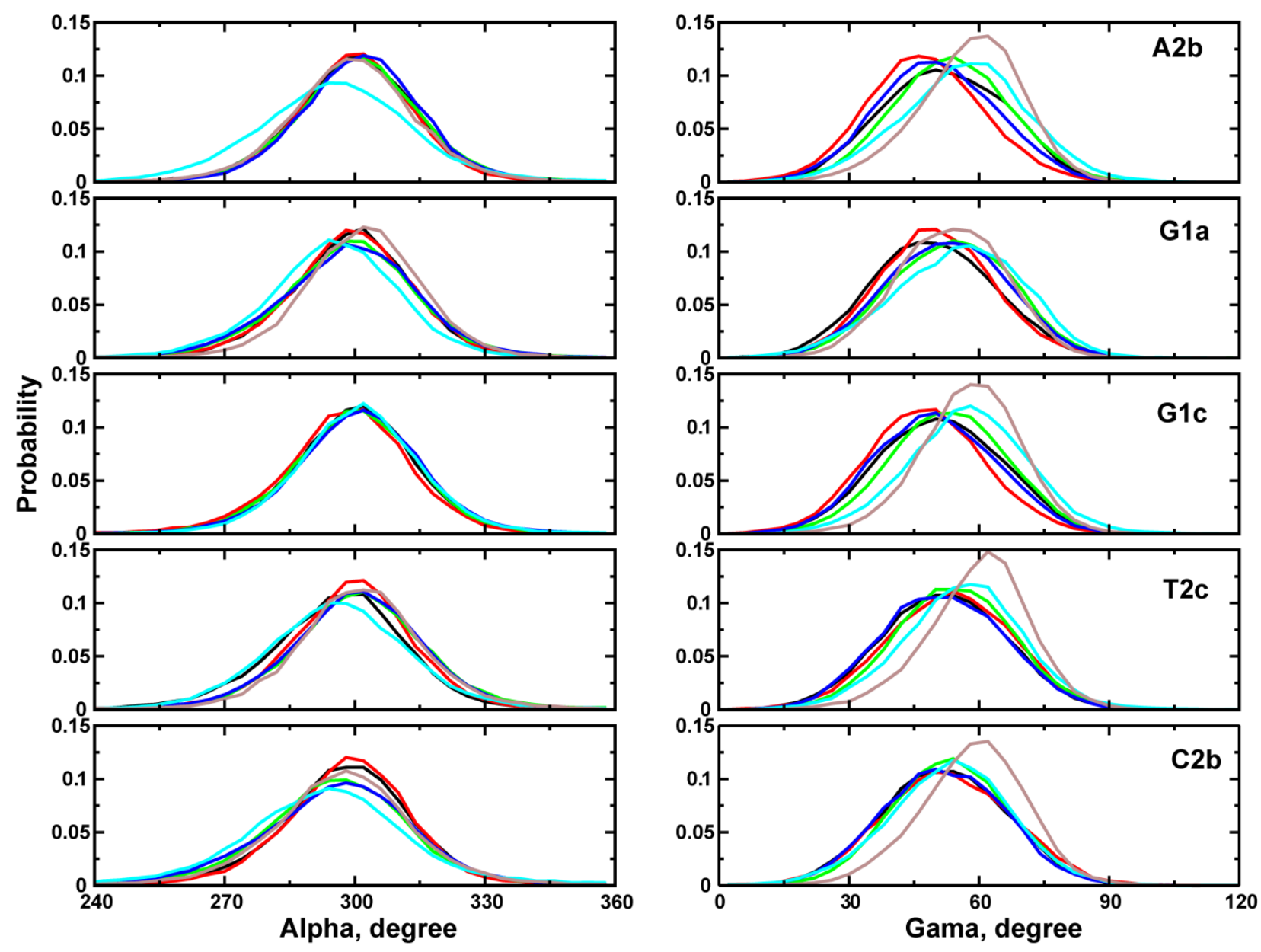

Figure S10: Probability distributions of backbone dihedral angles (in degree) corresponding to the urea nucleotides, its complementary nucleotide and their neighbour nucleotides in all the urea incorporated DNA duplexes. 

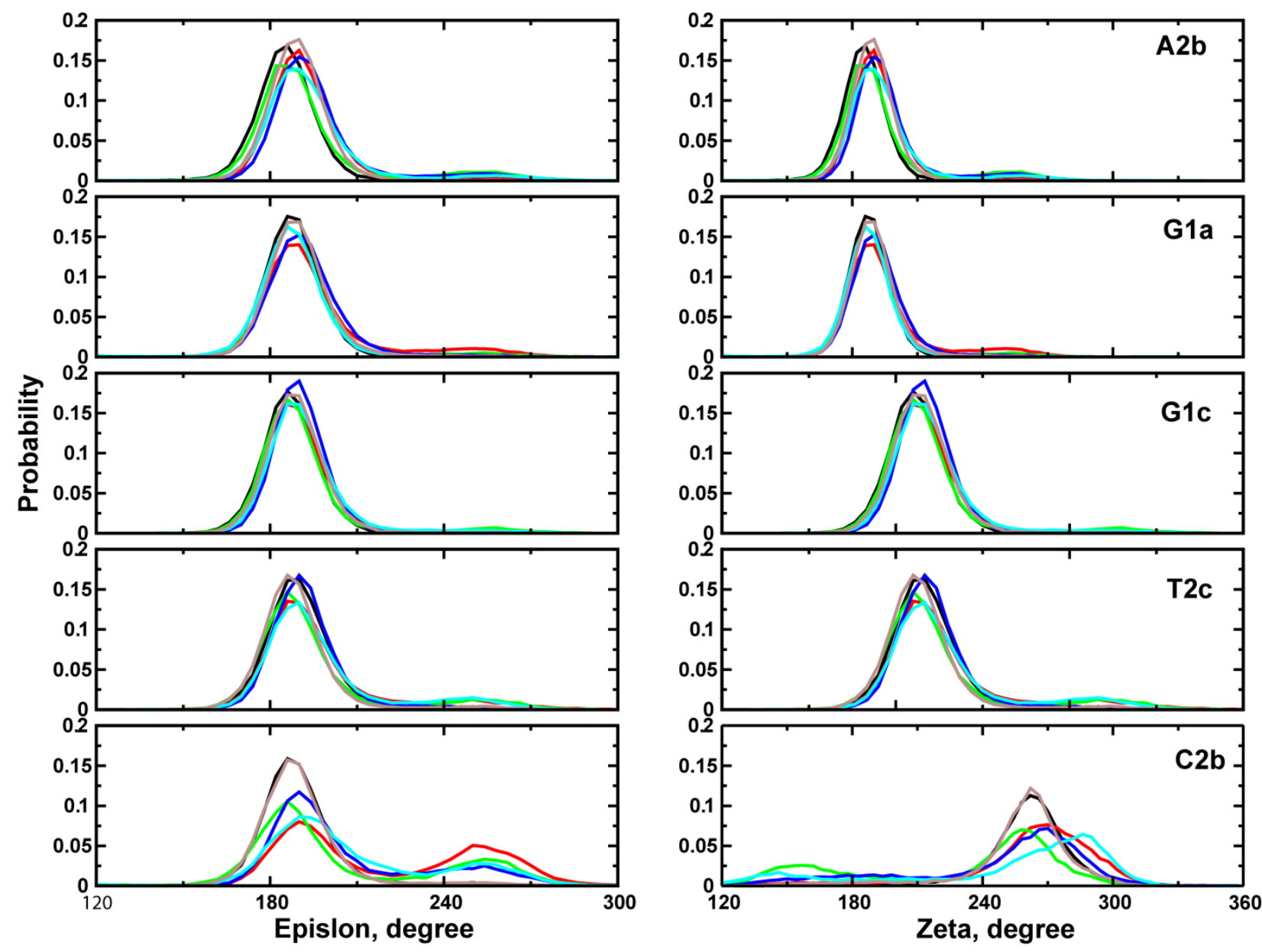

Figure S11: Probability distributions of phosphodiester bond angle (in degree) corresponding to the urea nucleotides, its complementary base and their neighbour nucleotides in all the urea incorporated DNA duplexes. 
Table S1: Interaction energies between urea and its complementary base and their neighbour AT and GC base pairs of all the urea incorporated DNA duplexes. The interaction energies corresponding to base pairs in pure DNA duplexes are also included. All the interaction energies are in $\mathrm{kcal} \mathrm{mol}^{-1}$. (See Figure 4)

\begin{tabular}{lccc}
\hline Duplex & cU-Urea & AT & CG \\
\hline (a) Adenine & & & $-22.60 \pm 0.02$ \\
B-DNA & $-10.83 \pm 0.01$ & $-10.84 \pm 0.01$ & $-22.48 \pm 0.01$ \\
A2b & $-7.34 \pm 0.12$ & $-10.73 \pm 0.01$ & \\
(b) Guanine & & & $-22.42 \pm 0.08$ \\
B-DNA & $-22.17 \pm 0.08$ & $-10.65 \pm 0.29$ & $-21.24 \pm 0.10$ \\
G1a & $-5.61 \pm 0.69$ & $-10.87 \pm 0.02$ & $-22.61 \pm 0.04$ \\
G1c & $-14.97 \pm 0.08$ & $-11.02 \pm 0.01$ & $-21.99 \pm 0.06$ \\
(c) Thymine & & & $-22.02 \pm 0.10$ \\
B-DNA & $-10.98 \pm 0.01$ & $-10.85 \pm 0.01$ & \\
T2c & $-4.47 \pm 0.52$ & $-10.79 \pm 0.02$ & $-22.69 \pm 0.07$ \\
(d) Cytosine & & $-11.08 \pm 0.01$ & $-22.07 \pm 0.22$ \\
B-DNA & $-22.77 \pm 0.01$ & $-10.76 \pm 0.03$ & \\
C2b & $-6.98 \pm 0.75$ & &
\end{tabular}

